\title{
Towards a Fourth Cosmology of Doctor-Patient Relationship: A Reflection on the Virtual Patient Community PatientsLikeMe
}

\author{
Konstantinos Bletsos, George Alexias, Charalambos Tsekeris
}

Department of Psychology, Panteion University of Social and Political Sciences, 136 Syngrou Ave., 176 71, Athens, Greece.E-mails: bletsos@gmail.com, galexias@panteion.gr, tsekeris@panteion.gr

\begin{abstract}
The aim of this paper is the study of an online patient community (Patientslikeme) and, specifically, of the impact of virtuality in the shaping of the patient-doctor relationship. Drawing from Kozinets's research paradigm, known as ethnography of the Internet (Netnography), we investigate the patients' attitudes toward the medical profession. Examining relevant data from the ALS forum, we set up three main axes of analysis: (a) the energetic versus passive stance towards doctors, (b) the evidence-based scientific knowledge versus alternative forms of knowledge, and (c) the positive versus negative attitude towards doctors and medical practices. The underlying theoretical hypothesis, i.e. whether the virtual community empowers patients over against the authority of the medical profession, is verified. Other interesting findings involve the value of evidence-based scientific knowledge, the intermediary role of the online community in building the patient-physician relationship, and the development of an alternative discourse towards the dominant medical discourse. All the above suggest that we have entered an era where the control of the patient is being transferred to another (digital) dimension, in which virtual communities play a crucial role.
\end{abstract}

Keywords: Virtual Communities, Online Research, Medical Power, Scientific Knowledge, Cyberbehavior, Sociology of Health and Illness, Cybermethods

Acknowledgement: This reflection presents personal views of the authors. It is a modified version of a paper presented at the Second Panhellenic Interdisciplinary Conference "Mental Health and Telematic Applications" (organized by obrela.gr), which was held October 10, 2012 in Athens, Greece.

Following the dramatic gradual loss or decline of "real" or "physical" human communities, the highly contentious and ill-defined cyber-conception of virtual communities, within the ICTsand-Society field, signifies a decisive historic break with material human geography and the subsequent emergence of dynamic Net-based "social aggregations" (Rheingold 1993), electronically-grounded, complex networks of interactive social, cultural, political and economic relations.

Virtual communities, originally anticipated by J.C.R. Licklider and R.W. Taylor as early as 1968, increasingly move towards the overwhelming creation of a global (or, more precisely, local/global or "glocal") virtual society, which optimistically promises unlimited/uninterrupted democratic freedom of speech and self-expression, as well as the general revision and revival of the so-called public sphere (as defined by J. Habermas): "The vision of a citizendesigned, citizen-controlled worldwide communications network is a version of technological utopianism that could be called the vision of "the electronic agora'" (Rheingold 1993: 14). This reflexively entails the innovative transdisciplinary conception of "digital citizenship" as a fundamental "capacity" to actively engage and participate in society, economy and politics online.

In that sense, an emerging virtual community, as an essentially anonymous (yet public) online community of common interests, visions, aspirations, tasks, duties, goals and orientations, is much "more than just an array of computer-mediated communication messages; it is 
a sociological phenomenon" (Matusitz 2007: 24). But, above all, it is an inherently transdisciplinary phenomenon.

Virtual communities emerge out of the life-worlds of online social interaction and organize themselves on the basis of common values, mutual participation, common interests (or friendship) and a sense of commitment and dedication (see Tsekeris and Katerelos 2012). To better understand the "value" of social interaction, it is expedient to carefully study how particular power relations are developed and cultivated within online environments.

Virtual communities are "virtual" in the sense that they operate within the cyberspace, without the occurrence of face-to-face communication or any other physical contact. But is this indeed a critical feature? For Kollock and Smith (1999), many traditional communities (e.g. ethnic or religious) identify themselves not so much through the physical presence of their participants, but mostly through shared experience, beliefs and values ${ }^{1}$.

Furthermore, it is customary that people surf the Internet with the aim to extract information and online support regarding their health (Diaz et al. 2002). Increasingly, this online search plays an important role in self-health education. For example, the survey of Pew Internet and American Life Project (Fox and Rainie 2002) discerned that 73 million Americans have searched for health information online.

The same study also found that a significant number of people have relied on online information to take important health decisions, while others used such information to make discussions with doctors (Broom 2005). Nevertheless, Diaz et al. (2002) argue that $59 \%$ of Internet users do not really discuss their online search results with their doctors. One possible reason for such patients' reluctance is that physicians may feel threatened and react negatively towards them (Anderson et al. 2003).

Virtual Patient Communities are emergent communities involving specific health problems. Members of these communities exchange views, experiences and opinions in relation to treatment programs, but also exchange strong feelings of social support and solidarity. However, there is a lack of considerable research documentation upon the effects of the virtual dimension in the everyday experience of illness (Broom 2005).

Several participants to Broom's (2005) research responded that the Internet helped them distance themselves from their illness and their symptoms. They found it much easier to share their experiences, when they perceived themselves to be somehow decoupled from their biological body. For Broom (2005), there is a noticeable difference between anonymity (mostly represented in negative terms) and disembodied communication, which is described as a situation that promotes the exchange of experiences, intimacy and mutual support. In each case, there are many important questions awaiting answers (Broom 2005, 340):

- Does the Internet change the way patients perceive their disease?

- What is the role of the Internet to self-health education?

- Does the Internet encourage the critical interrogation of the medical knowledge, thus

affecting the process of decision-making on important clinical issues?

- Does the Internet influence the dynamic doctor-patient relationship, and if so, how do doctors face this change?

Buckland and Gann (1997) argue that the Internet is powerful enough to allow for the patient's crucial move from the role of the passive receiver/consumer to the active role of researcher. The search for information and, in general, the process of converting the patient to an expert is a painful and often dead-end process, to the extent that patients possess neither the necessary skills nor the necessary time.

On such basis, it is not surprising to note that most patients consult their doctors to receive guidance and support, thus strengthening the medical authority and the power this generates (Anderson et al. 2003). According to Broom (2005), the Internet can possibly em-

\footnotetext{
${ }^{1}$ As Shaffer and Anundsen (1993) perceptively observe, a human community, as a dynamic whole, emerges when a group of people sharing common practices identify itself as something more than the sum of its members and takes a good long-term commitment to a collaborative way forward. The most characteristic example of this process is the development of trust, defined as an expectation of honest and cooperative behavior, based on shared norms among community members (Fukuyama 1995).
} 
power patients towards a sense of control over the disease. Broom borrows the etymology of Roberts (1999), whereby the patient empowerment refers to a new role characterized by modulation of power in the doctor-patient relationship.

By gaining more control over issues regarding their health and their relationship with health professionals, patients now have the ability not only to get information pertaining to their disease, but also to evaluate the skills of their doctors in comparison with the skills of other physicians, as they are presented by members of (self-organized) virtual communities. Interestingly, Gibson (1991) indicates that the empowerment of the patient can be characterized:

a. As the reduction or absence of sensation, or loss of power.

b. As the reduction or absence of feeling, or of helplessness and lack of hope, a sense of alienation, victimization or harness.

The Internet is a valuable source of information for patients seeking better control over their treatment. This means that patients discover different choices in their treatment and, as a result, reduce their dependence on the opinion of experts. Although most patients believe that Internet use does not necessarily affect treatment decisions (or treatment outcomes), they admit that it has offered great help in the process of decision making, reducing the uncertainties behind the alternative choices (Broom 2005, 333).

Broom (2005) argues that, contrary to the fears of the medical community, online information search do not increase the negativity or skepticism of patients in relation to biomedical (conventional) treatments. But the choice between different (classical) treatment options, increase the bargaining power, since the patient presents any clinical choice as a result of his/her informed decision. The informed patient often moves beyond the typical patient role. As a consequence, this is likely to raise resistance from the physicians, while the feeling of rejection creates anxiety, confusion, irritability to patients, and communication barriers (Broom 2005, 334).

Since the late 1990s, hundreds of scientific articles, in disciplines from Medicine to Sociology of Health and Illness, point out that the Internet creates a new kind of patient: an informed patient, responsible for his/her own health and willing to make rational decisions. In this regard, the empowerment comes through rational information and is used to direct attention from the doctors to the personal experience of illness (Segal 2009, 357).

An important aspect emerging from the recent research on virtual patient communities is the relationship between the body image and the development of trust among members. In specific, Broom (2005) found that many of the patients felt that the Internet helps them move away from disease and symptoms, as well as that it is much easier to share their experiences in the virtual environment, which somehow gives them the feeling of being freed from their bodies.

Patients seem to be aware of the difference between the anonymity of the Internet (represented as something negative) and disembodied communication, experienced as a positive situation in which the individual is ready to share his/her experiences. This separation between the embodied and the disembodied self helps some patients to open up to others, seeking information and support. Hence, disembodiment allows for a controlled transition to intimacy and mutual support (Broom 2005, 340).

Founded in 2004, the virtual community of patients PatientsLikeMe (PLM) is currently offering fourteen different communities that specialize in similar disease entities, in which approximately 47,000 patients interact, using social networking tools similar to those used in Facebook, as well as health management tools similar to those existing in Personal Health Records (PHR).

The general objectives of the community, however, are not entirely clear since they are related to the provision of anonymous data for use in pharmaceutical industry and medical technology companies. This relationship between patient-community and the desire to (at least partially) a profit through participation, call into question the motivation behind the design of websites, such as PLM. In addition to general social networking tools, such as user profiles, comments and personal messages, PLM provides specialized tools that al- 
low patients to monitor and distribute relevant information, such as symptoms, treatments, and medical data.

These features allow users to compare their own experiences with the experiences of other patients while enabling them to take a more active role in determining treatment options in collaboration with the providers of care. Users can choose from a range of options concerning privacy, but the community emphasizes the "philosophy of open data" (Openness Philosophy), a statement that supports the democratization of patient data via the Internet, in order to "accelerate research like never before" (Brubaker et al. 2010).

Access to patient data is a key issue in medical research and treatment. Modern technologies offer the possibility of enormous data concentration for epidemiological studies. An important difference between PLM and other similar systems is based to the phenomenon called relative sensitivity of stored and shared data. In most cases, the legal restrictions that protect the privacy of patients also restrict the availability of data, both for patients and for other stakeholders. The solution offered by PLM in this problem requires serious consideration. The website (www.patientslikeme.com) has an extensive and strict policy on privacy, coupled with a philosophy of openness. By allowing patients to record medical data, PLM avoids the limitations that exist in other systems of medical record data (Brubaker et al. 2010).

PLM uses a sophisticated system of data entry, which utters the possibility of visualizing. This system serves two main purposes:

- It allows patients to enter and track their personal data by means of sophisticated graphics. As argued by the Frost and Massagli (2008), this system is based on the project "the image as data", a process whereby "through the collection, analysis and understanding of the data, patients can understand the value of daily behavior in health promotion".

- It gathers and sells the data for profit and promotion of medical research and related services.

In this respect, the data entry system has to balance between the patients' need for a useful website and market demands for (qualitative) data. The PLM extensively meets the demands of patients. For example, the recording system allows patients to choose to monitor symptoms they consider important, even gives the possibility for customization of symptoms. This configuration, however, creates problems in patient data. Smith and Wicks (2008) showed that patients ignore medical taxonomies and use their own classifications (folksonomies).

Data frequently include idioms or unstructured data, which represent a number of things that only make sense within that framework. Although people who read these data can relatively easily make sense, the computational data processing is still a challenging process. In this sense, the need of researchers (and companies) to structured information can be found in conflict with the need of patients to offer non-customized and unstructured data. Clinicians, researchers and manufacturers of medical products that receive data from the community are often faced with serious questions concerning the validity and reliability of this data:

1. Patients may not be accurate in self-reports.

2. A patient who is willing to contribute relevant data is probably not representative of all patients.

The present analysis that focuses on this specific community of patients, refers to patients with high educational level who have Internet access and relevant technological skills. This probably is not representative of the entire population of patients suffering from the same disease (Brubaker et al. 2010).

The research paradigm was based on Netnography (Kozinets 2002). In October 2010, we were registered on the community forum of patients suffering $\mathrm{ALS}^{2}$. For access to research data we used keywords (key searches), which considered being relative to our hypothesis. This was followed by a systematic study of the posts in the community forum and of

\footnotetext{
${ }^{2}$ Acute Lateral Sclerosis (ALS) or Lou Gering Disease is a chronic degenerative disease of motor neurons of the central nervous system, causing progressive loss of mobility of the muscles and leading to serious disability and death on average 2-5 years from diagnosis.
} 
the relevant activities (articles in magazines and newspapers, newsletter, mail alerts from Google Scholar, videos etc) of PLM, which aimed to enforce our understanding of social experiences and subjective meanings beyond the obvious interactions. To answer our research hypothesis, we investigated the statements of patients using axes which are constructed on the basis of differentiation (distinction) between attitudes ${ }^{3}$ related to:

- The active or passive role toward doctors.

- The value on documented (prevailed) scientific knowledge as opposed to the emergence of new or alternative therapeutic approaches.

- Patients beliefs about doctors (and medical practices), as articulated in cognitive and emotional dimension.

The characteristics of the virtual environment (anonymity, constant communication, speed, communication flow, interaction, easy access to expertise, support networks etc) offer the possibility of shaping a new form of relationship between patient-physician (characterized by reversals in the classical form of power relationships), which was the result of the dispute in possession of scientific knowledge. Access to scientific knowledge and interaction between patients in the community shape a new type of patient-physician relationship characterized by:

- Upgrading the patient role as an equal member of the treating team.

- The shift to scientific evidence and documentation.

- The patient "activation" in relation to issues of health and disease.

- At the formation of powerful information and support networks.

In this analytical context, the basic theoretical hypothesis is as follows: The PLM helps patient empowerment toward an active role characterized by:

1. The search for information in relation to the disease through strong community (sociotechnological) networks.

2. The emergence of a patient discourse against the dominant medical discourse.

3. The critical stance against doctors and medical practices.

PLM promotes patient empowerment through the provision of knowledge (scientific and empirical) and support to its members. At the same time, community constructs "reality" by the mean of interpretation of complex phenomena (such as sickness and knowledge) and guidance of members' attitudes (e.g. attitudes towards doctors). With the adoption of a critical stance (against knowledge and health professionals), PLM promotes the patient emancipation towards the shape of an autonomous (or alternative ${ }^{4}$ ) discourse. These phenomena are dynamically formatted by:

a. The collection of chaotic and scattered scientific knowledge within cyberspace.

b. The interpretation and explanation of phenomena that would otherwise be obscure (popularization of science), which leads to a system of representations in relation to "what is illness", but also how to establish the patient's identity.

c. The (digital) representation of experience and comparative approach (as compared to the history of the patient herself but also to the stories of others) through a system of normalization that serves as evaluator and as a regulator of patient expectations.

d. The emergence of a patient discourse against the dominant medical discourse.

e. The critical stance against doctors and medical practices.

In these ways the PLM community constructs and reconstructs knowledge that is both empirical and scientific. The reconstruction of knowledge refers to research and scientific articles that members use to explain situations that are transported through the exchange of experiences. On the other hand, the construction of knowledge refers both to the experiential knowledge of members and scientific knowledge extracted through independent clinical trials of pharmaceutical agents (e.g. lithium), the publication of scientific articles, and the provision of patient data to pharmaceutical companies and research centers.

\footnotetext{
${ }^{3}$ We use "attitude" in the sense of readiness for action.

${ }^{4}$ It seems to be alternative rather than autonomous, as far as the patient discourse is based on dominant scientific knowledge.
} 
The complex and largely contradictory elements that make up the PLM community are not easily interpretable within the bounds of linearity and Cartesian dualism ${ }^{5}$. For example, the organizational model of community is composed of two distinct, interrelated levels, which cannot be analyzed separately, but always inside the complex techno-social environment of virtuality. These two levels place members in different positions, according to the dynamic balance of dominating forces (Members-Administrators), but also produce different orders of techno-social phenomena.

To analyze these two levels of community formation, we used the sociological approach of ANT (Actors Network Theory) ${ }^{6}$. Following this anti-essentialist theory, the potential emergence of virtual communities is presented as an active ongoing process of constructing a network of associations in which human and non-human entities (actants) mutually obtain existence as events in a socio-technical imaginary (see Law and Hassard 1999; Tsekeris 2008).

According to the above, the community consists of two distinct ${ }^{7}$ sub-networks and extends into multiple dynamic spatial and temporal relations. We describe the first level as organizational. At this level, the role of actants is energetically performed by humans and nonhuman parts of the network (artefacts) behind the PLM (owners, scientists with an employment relationship with the community, software developers, managers, corporate employees, etc).

The subnet mostly produces top-down phenomena, since it is the design center and the center that emanates the policies which govern the relationships both within the wider network and with external networks. This is the birth place of policies concerning the rights and obligations of members, permissible and non permissible behaviors, capabilities and limitations of the software, privacy rules, relationships with pharmaceutical companies and, in general, all the profits, policies and scientific construction of PLM.

Unlike the other subnetwork, the patient's network consists of community members ${ }^{8}$ who establish social networks of strong emotional relationships and produce emergent phenomena - a kind of social dynamics driven from below (bottom-up approach). The ANT approach indeed helps us to meticulously study complex socio-technological phenomena, but it is relatively inefficient to substantially explain behavior as the structural upshot of the different positions held by members on both subnets (members-administrator).

A better understanding of social interactions requires analytical reference to the whole framework determined by power differences between members and administrators of PLM. The twin system of member award ${ }^{9}$ is a possible example. Although in terms of design both systems are derived from the organizational subnetwork, they produce an entirely different class of social dynamics:

1. The system of stars reflects the social control exercised by PLM policies to community members, through consistent rewards to members who comply with the requirements (see Figure 1).

2. The system of helpful marks on the opposite used by members to reward the post that helped them. This system reflects the emerged phenomena produced by the interaction of members, and thus are totally outside of administrator's control. An interesting phenomenon at that level becomes the resistance to the requirements of the PLM by some members with particularly strong presence (hundreds or thousands of helpful

\footnotetext{
${ }^{5}$ That means, the philosophical logic pertaining to a world of purity, unity, homogeneity and predictability. This also assumes proportional relationships between the cause and the effect, something that by definition excludes any alternative explanation.

6 A good amount of literature on Actors Network Theory can be found in this hyperlink: http://www.lancs.ac.uk/fass/centres/css/ant/ant.htm\#ear (Accessed September 14, 2012)

${ }^{7}$ Distinct but in no case separate from one another, to the extent that they mutually interact to produce multiple relations, in a higher spatial dimension of spiral technosociological phenomena - a process of "translation" according to ANT terminology.

${ }^{8}$ And nonhuman parts as well, like interconnected computers.

9 The twin system of member awards is referred to: (a) the system of stars in which the PLM awards the members according to the quality of data provided; (b) the system of helpful marks in which the community members award each other according to the usefulness of their forum posts.
} 
marks) which blatantly refuse to comply informing the (market oriented) data (0-1 stars).

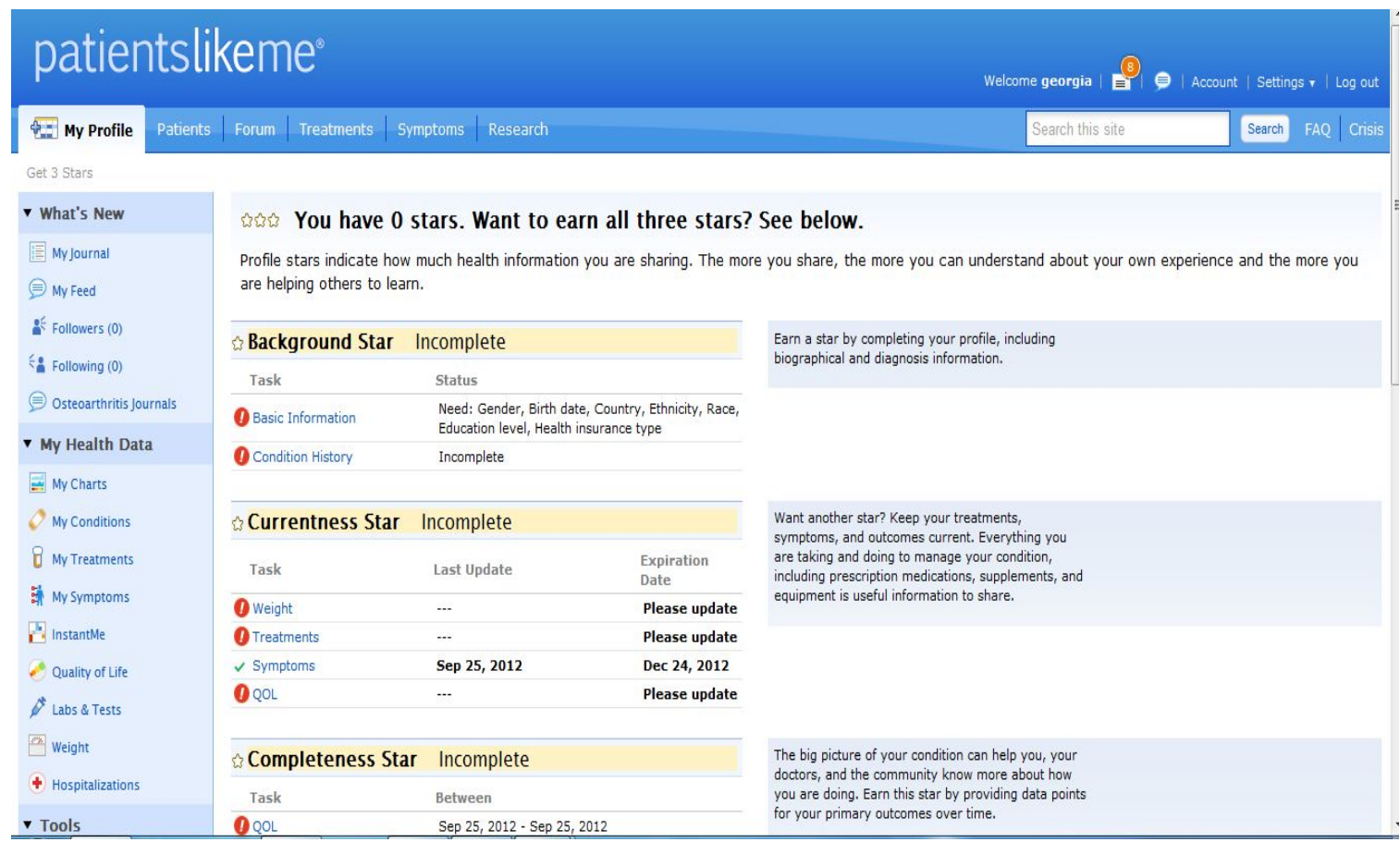

Figure 1: The system of stars in PLM (PatientsLikeMe 2012)

This observation is important because, according to Stalder (1997), the coherent or the solvent component of a network depends on power relations developed within (but also in relation to) competing networks. But virtual communities do not operate in a social vacuum, to the extent that emergent technosocial phenomena acquire meaning when transferred to the (non-virtual) patients' life. The community's influence on shaping doctor-patient relationship requires analytical reference to the power-loaded context that defines differences in the possession of knowledge between doctors and patients. The re-organization of this relationship goes through:

- The development of a critical stance towards doctors and medical practices, by means of representations of what constitutes the "good doctor" (e.g. the relevant thread titled as Rate Your Neurologist).

- The emergence of knowledge as a determinant of patient empowerment.

- The emotional support and mutual aid among members.

In summary, we argue that the virtual community PLM plays an important mediating role in the traditional unbalanced relationship between doctors and patients. To achieve this, PLM creates mechanisms of production and concentration of dispersed knowledge, but also offers an ideological framework as the matrix of representations of complex phenomena such as knowledge, health, illness, patient, doctor etc. From a historical perspective, it is obvious that we have entered a wholly new era where the control of the patient is being transferred to another (digital) dimension, in which virtual communities obtain a crucial role. This era could also be the beginning of the fourth cosmology, according to Sarah Nettleton's taxonomy $(2009)^{10}$

\footnotetext{
${ }^{10}$ According to Nettleton (2009), the first cosmology of doctor-patient relationship, from 1770 to 1800 , was "bedside medicine" when doctors and patients had a close personal relationship. With the patient being the employer of the physician, s/he had great influence on doctor's theories for the disease. The second cosmology, from 1800 to 1840, was "the hospital medicine" as the patients had been massively hospitalized. The control was hereby transferred from patients to doctors, who developed a coherent theory of the illness, based on the detected pathology. Finally, according to the third cosmology, the medical knowledge has gradually been fostered by the "laboratory medicine" as scientists used to monitor the diseases through lab tests.
} 


\section{References}

Anderson, James, Michelle Rainey and Gunther Eysenbach. 2003. The impact of cyberhealthcare on the physician-patient relationship. Journal of Medical Systems 27 (1): 67-84.

Broom, Alex. 2005. Virtually He@lthy: The impact of Internet use on disease experience and the doctor-patient relationship. Qualitative Health Research 15 (3): 325-345.

Brubaker, Jed, Caitlin Lustig and Gillian Hayes. 2010. PatientsLikeMe: Empowerment and Representation in a Patient-Centered Social Network. Accessed September 11, 2012. http://www.gillianhayes.com/wp-content/uploads/2011/01/CnP11 PatientsLikeme.pdf

Buckland, Sarah and Bob Gann. 1997. Disseminating treatment outcomes information to consumers: Evaluation of five pilot projects. London: King's Fund Publishing.

Diaz, Joseph, Rebecca A. Griffith, James J. Ng, Steven E. Reinert, Peter D. Friedmann and Anne W. Moulton. 2002. Patients' use of the Internet for medical information. Journal of General Internal Medicine 17 (3): 180-185.

Fox, Susannah and Lee Rainie. 2002. Vital decisions: How Internet users decide what information to trust when they or their loved ones are sick. Washington, DC: Pew Internet \& American Life Project.

Frost, Jeana and Michael Massagli. 2009. PatientsLikeMe the case for a data-centered patient community and how ALS patients use the community to inform treatment decisions and manage pulmonary health. Chronic Respiratory Disease 6 (4): 225-229.

Fukuyama, Francis. 1995. Trust: Social Virtues and the Creation of Prosperity. New York: Free Press.

Gibson, Cheryl. 1991. A concept analysis of empowerment. Journal of Advanced Nursing 16 (3): 354361.

Kollock, Peter and Marc Smith. eds. 1999. Communities in Cyberspace. London: Routledge.

Kozinets, Robert. 2002. The Field Behind the Screen: Using Netnography For Marketing Research in Online Communities. Journal of Marketing Research 39 (847): 61-72.

Law, John and John Hassard. eds. 1999. Actor Network Theory and After. Oxford: Blackwell.

Matusitz, Jonathan. 2007. The implications of the Internet for human communication. Journal of Information Technology Impact 7 (1): 21-34.

Nettleton, Sarah. 2009. Commentary: The appearance of new medical cosmologies and the reappearance of sick and healthy men and women: a comment on the merits of social theorizing. International Journal of Epidemiology 38 (3): 633-636.

Rheingold, Howard. 1993. The virtual community: homesteading on the electronic frontier. Reading, MA: Addison-Wesley.

Roberts, Kathleen. 1999. Patient empowerment in the United States: A Critical commentary. Health Expectations 2 (2): 82-92.

Segal, Judy. 2009. Internet Health and the 21st-Century Patient: A Rhetorical View. Written Communication 26 (4): 351-369.

Shaffer, Carolyn and Kirstin Anundsen. 1993. Creating community anywhere: finding support and connection in a fragmented world. New York: Putnam Pub Group.

Smith, Catherine and Paul Wicks. 2008. PatientsLikeMe: Consumer health vocabulary as a folksonomy. AMIA Annual Symposium Proceedings 2008: 682-686.

Stalder, Felix. 1997. Actor-network theory and communication networks. Accessed September 25, 2012. http://felix.openflows.com/html/Network Theory.html

Tsekeris, Charalambos. 2008. Exploring the Social in Science and Technology Studies. The Social Sciences 3 (7): 521-524.

Tsekeris, Charalambos and loannis Katerelos. 2012. Web 2.0, Complex Networks and Social Dynamics. Contemporary Social Science: Journal of the Academy of Social Sciences 7 (3): 233-246.

\section{About the Author}

\section{Konstantinos Bletsos}

is a psychologist and psychological researcher, graduated from the Department of Psychology, Panteion University of Social and Political Sciences, Athens, Greece. His research interests include cyberpsychology, psychological counseling, the sociology of health and illness, Internet addiction and virtual communities. E-mail: bletsos@gmail.com

George Alexias 
is Assistant Professor at the Department of Psychology, Panteion University of Social and Political Sciences, Athens, Greece. His research interests include the sociology of the body, medical sociology, the sociology of genetics and cybersociology. E-mail: galexias@panteion.gr

Charalambos Tsekeris

is currently lecturing at the Department of Communication and Media Studies, National and Kapodistrian University of Athens, Greece. He is a Senior Researcher at the Laboratory of Virtual Reality, Internet Research \& E-Learning, Department of Psychology, Panteion University of Social and Political Sciences, Athens, Greece. His research interests mostly involve human complex systems, psychosocial studies, virtual communities, epistemology and cybermethods. E-mail: tsekeris@panteion.gr 